\title{
Applications of Blockchain Technology in Logistics and Supply Chain Management-Insights from a Systematic Literature Review
}

\author{
Moritz Berneis *, Devis Bartsch and Herwig Winkler *(D) \\ Production and Operations Management, Brandenburg University of Technology, 03046 Cottbus, Germany; \\ Devis.Bartsch@b-tu.de \\ * Correspondence: moritz.berneis@b-tu.de (M.B.); winkler@b-tu.de (H.W.); Tel.: +49-(0)-355-69-4089 (H.W.)
}

Citation: Berneis, M.; Bartsch, D.; Winkler, H. Applications of Blockchain Technology in Logistics and Supply Chain Management-Insights from a Systematic Literature Review. Logistics 2021, 5, 43. https:// doi.org/10.3390/logistics5030043

Academic Editor: Robert Handfield

Received: 26 March 2021

Accepted: 17 June 2021

Published: 30 June 2021

Publisher's Note: MDPI stays neutral with regard to jurisdictional claims in published maps and institutional affiliations.

Copyright: (c) 2021 by the authors. Licensee MDPI, Basel, Switzerland. This article is an open access article distributed under the terms and conditions of the Creative Commons Attribution (CC BY) license (https:/ / creativecommons.org/licenses/by/ $4.0 /)$.

\begin{abstract}
The most successful applications of Blockchain Technology are still in the area of cryptocurrencies, although both scientists and practitioners have discovered the potential of Blockchain Technology in Supply Chain Management. There is a significant theoretical literature on Blockchain Technology, but there exists a lack of published case studies and concrete examples. This paper discusses whether this shortcoming is due to insufficient added value of the technology and identifies other possible reasons. Furthermore, this paper introduces Blockchain Technology, describes the origins of Bitcoin, the structure and core properties of the Blockchain, and examines smart contracts. A comprehensive and structured literature analysis identifies concepts for the use of Blockchain Technology in logistics in terms of economic benefits. Additionally, a cluster analysis regarding the topics of the relevant literature was conducted. One finding of the study is that Blockchain Technology is particularly worthwhile for goods with a high value. Moreover, if the trade volume of the respective goods is low, the advantages of BCT are maximized. At the same time, the demand for transparency and immutability of data must be more important than the protection of sensitive data. In addition to concrete use cases of Blockchains, an exemplary logistics process will be presented within the Luxury Supply Chain, showing the advantages of Blockchain Technology for each individual process step.
\end{abstract}

Keywords: Blockchain Technology; Smart Contracts; logistics; Supply Chain Management

\section{Introduction}

In 2008, an individual using the pseudonym Satoshi Nakamoto published the basic concept of the first crypto-currency, Bitcoin [1]. Bitcoin is a cryptocurrency, which does not require a central institution such as a central bank or a trusted third party, like PayPal. The Bitcoin network is based on Blockchain Technology (BCT) [2] (p. 22). The Blockchain (BC) is a distributed peer-to-peer database that consists of a network of equal nodes [3] (p. 1). Because the data is tamperproof and firmly integrated into the $B C$, the $B C$ can reduce human intermediaries. Currently, BCT is mainly used in the field of crypto-currencies, but researchers and companies have recognized the potential of BCT for other areas, such as logistics [4] (p. 263). This paper examines the application of BCT in logistics with the aim of identifying its economic benefits and practical applications.

With globalization, logistics is confronted with new challenges stemming from a worldwide network that has to be planned, guided, optimized and controlled. This network consists of a multitude of different companies, which are currently controlled individually with different central systems [5] (p. 19). Holistic controlling of the Supply Chain (SC) could increase its transparency and both generate and maintain competitive advantages over other SCs [6]. Logistics and Supply Chain Management (SCM) could benefit tremendously from BCT's decentralized structure, high transparency and security against manipulation [7] (p. 7). The following comprehensive literature analysis demonstrates the growing interest of logistics and SCM in BCT. The aim of the paper is to answer the 
following two research questions: (a) What is the international state of research regarding BCT in SCM?; (b) Which areas of SCM are covered by current publications? Furthermore, the paper aims to provide insights into selected case studies and real world examples.

\section{Fundamentals of Blockchain Technology}

\subsection{Origin of the Blockchain Technology}

In 2008, during the global economic crisis that began in 2007, and presumably triggered by the bursting of the speculative bubble of the American real estate market, the pseudonymous Satoshi Nakamoto published the concept for Bitcoin in a white paper [1]. The Bitcoin network is very similar to conventional payment services, such as PayPal, in terms of its functionality for the user [2] (p. 22). However, Bitcoin has little in common with traditional currencies, which can be available in digital form through financial services providers such as PayPal. When digital currencies rely on a central node, a hostile attack can cause serious damage [8] (p. 1).

Bitcoin, on the other hand, has a decentralized structure. With a public BC, anyone can become a user of the network. Since the network is stored on many devices worldwide, an attack on the network is almost impossible [9]. Time has proven this, as the Bitcoin network has been online almost continuously without major problems since its launch in early 2009. The market capitalization reached a peak of around USD 300 billion in 2017 (See: coinmarketcap.com (last accessed on 10 June 2021)), which has aroused public interest and attracted the attention of new investors.

Bitcoin's enabling technology is the BCT. However, Bitcoin cannot exist without the $B C$ and is inseparably linked to the $B C$ [2] (p. 26). The BC has enormous potential for largescale improvements in many different areas [2] (p. 22). This is a new type of decentralized data structure that enables the unique properties of the Bitcoin network. The following section describes the basic structure of a BC and explains its most important properties and key features.

\subsection{Basics and Structure of the Blockchain}

$\mathrm{A} \mathrm{BC}$ is, as the name implies, a chain of blocks. Each block has an identical structure, consisting of a head and a body. Each block contains an exact reference to the previous block, so that a chain is formed (see Figure 1). The only exception is the so-called Genesis block. This is the first block of the BC, defined by a software. All transactions are combined and stored in the next block, a process that is called mining [9] (p. 244).

The header of a block contains the obligatory reference to the previous block, as well as other information, such as a timestamp and the version of the block. To make the blocks uniquely identifiable, they are assigned a unique name by an algorithm. The reference to the previous block results in a chain of blocks that becomes longer and longer. The deeper a block is contained within this chain, the more participants have to be manipulated for a change, which reduces the probability of manipulation. The most important feature of the $\mathrm{BC}$ is that the previous blocks cannot be changed without having to rebuild the entire $\mathrm{BC}[10]$.

The main part of each block is the body. All transactions that did not make it into the last block are transferred into the current block. The size of each block is fixed, and therefore the number of transactions per block is strongly limited. In the body, these transactions are stored, as well as all related information. In the Bitcoin example, this is the data about the sender, the recipient and the amount in Bitcoin [11] (p. 561). 


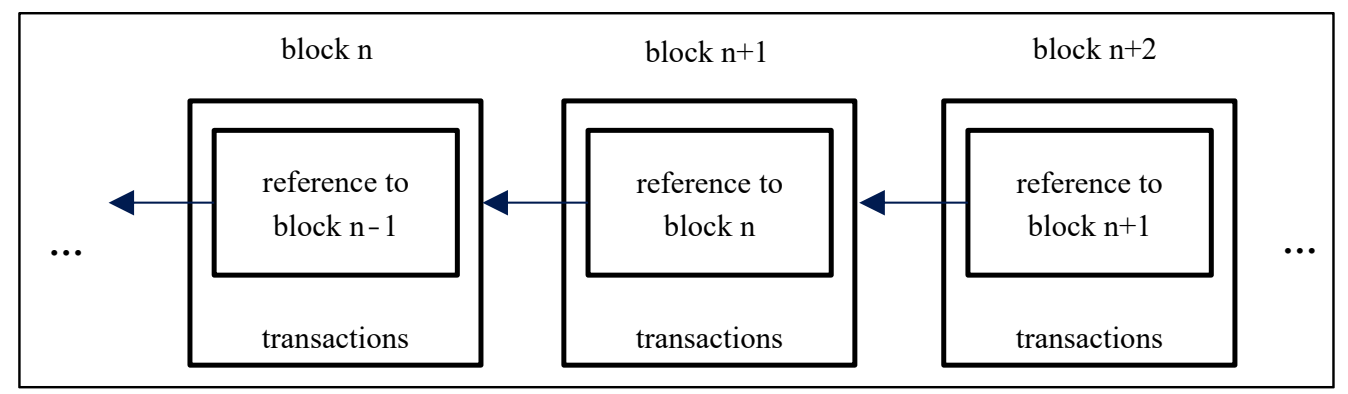

Figure 1. Schematic representation of the structure of a Blockchain [12] (p. 2293) (slightly modified).

As mentioned above, the $\mathrm{BC}$ has a decentralized structure and is not based on a central server or a central institution. Instead, the BC consists of a network of so-called nodes. Each user can keep a complete copy of the $\mathrm{BC}$ and check the new blocks. The more nodes that carry the network, the more copies there are of the $\mathrm{BC}$ and the more secure the network becomes. However, for a strong unique network, there must also be agreement on the BC. This is achieved by so-called consensus mechanisms; the BC of Bitcoin uses the Proof-of-Work (PoW) algorithm [11] (p. 559).

The PoW algorithm consists of a mathematical problem. In the example of Bitcoin, the first correct solution to the problem will be rewarded with Bitcoin and published, together with the new confirmed transactions. The solution can be verified very quickly by all other nodes and, if correct, can be attached to the $\mathrm{BC}$, including the transactions. An alternative to the PoW algorithm is the Proof-of-Stake (PoS) algorithm. Here, the miners are liable for the authenticity of a block with a part of their assets. Since no complex mathematical problems have to be solved, this algorithm is much more power-efficient and scalable. This means that the network is cheaper to operate, but, on the downside, the security of the network is lower than with the PoW algorithm [13] (p. 3).

\subsection{Key Features of the Blockchain Technology}

The $\mathrm{BC}$ is structured as described above to enable the most important feature of BCT, the decentralized structure. This is a very important security feature, because all changes have to be adopted by all participants of the network. Since each participant is allowed to hold a copy of the $\mathrm{BC}$, and there is consensus on the $\mathrm{BC}$ between the participants, the transactions in the previous blocks are unchangeable. This transparency means that all transactions can be checked by all participants. The more nodes the network has, the more fail-proof it is; in the case of Bitcoin, even satellites (See: blockstream.com/satellite/ (last accessed on 10 June 2021)) are a part of the network [14] (p. 2).

The specific structure of the $\mathrm{BC}$ and the high level of security gained also has its price. Each node holds every transaction ever made, must validate new transactions and must attach them to the stored BC. This very inefficient procedure (compared to conventional databases), in addition to the limited block size, results in very limited scalability. The Bitcoin network can process approximately seven transactions per second (TPS) [11] (p. 561). A centralized payment network like Visa claims to be able to process up to 65,000 TPS (See: Visa Factsheet. https://usa.visa.com/dam/VCOM/download/corporate/media/ visanet-technology / aboutvisafactsheet.pdf (last accessed on 10 June 2021)). Scalability can only be improved at the expense of decentralization or security. To improve the scalability, consensus mechanisms can be used with less security or they can be switched to private, permissioned BCs [15] (p. 6). Another problem is the dilemma between the highest possible transparency and the highest possible data protection. In the example of Bitcoin, the transactions are transparently visible to every participant in the network, which is necessary for a complete documentation of the ledger. Each Bitcoin can be traced back to its creation, and the true identity of the Bitcoin owner is often revealed at the interfaces to reality, e.g., the exchanges. Further technical challenges and barriers in implementing the technology are discussed in Section 4.2 [13] (p. 7). 
In summary, the BCT is a decentralized, transparent data structure and therefore it is also a very secure data structure. It functions without a central control system and stores information, such as transactions or programs, in chained blocks [16] (p. 5).

\subsection{Smart Contracts}

Currently, contracts are drafted by lawyers according to current law, in the case of disputes the contracts are judged by the judges of courts. If necessary, the executive power then enforces the contracts [17] (p. 265). Smart Contracts do not need all this, they enforce themselves independently according to previously defined, unambiguous rules. With Smart Contracts, the program code is stored on the BC. Smart Contracts are contracts because they are an agreement between two or more parties and contain fixed conditions. They are smart because the Smart Contract automatically recognizes which case of the contract has occurred and then automatically carries out the contractually agreed actions for this case. In most cases, these actions are transactions in which a pre-determined amount is sent to a specific address. Ethereum is measured by market capitalization, the second largest crypto-currency, and is therefore the most famous public BC supporting Smart Contracts [18] (p. 93).

The following example, based on the work of Asadi Bagloee and colleagues (2019), is intended to illustrate the principle of Smart Contracts, by means of a bet on the weather between two friends. Neither of the two friends knows what the weather will be like tomorrow, and they agree that the winner gets $€ 100$ from the loser. The two friends could hire an unbiased, trustworthy third party, like a lawyer, which they do not want. A Smart Contract can solve the bet cheaply and easily. First of all, it is defined at which weather conditions who wins and where the Smart Contract should get the weather information from. Then both deposit $€ 100$ into the Smart Contract and leave their addresses. The Smart Contract automatically determines the winner at the agreed time and initiates the corresponding transaction [19] (p. 3).

Nick Szabo already developed the idea of automated contracts in the 1990s [20]. Prior to the BCT, there was no suitable secure technology on which Smart Contracts could have run safely. The core characteristics of the BCT mentioned above enable unchangeable, decentralized and therefore secure, Smart Contracts. In summary, Smart Contracts can automate payments between two parties and ensure that the two parties do not have to trust each other, because both can trust the Smart Contract, due to its predictable behavior. Smart Contracts can provide higher security at lower costs. Therefore, Smart Contracts are assigned an important role in the application of BCT in SCM [16] (p. 21), [21] (p. 21).

\section{Structured Literature Analysis}

The comprehensive and structured literature analysis was conducted in six steps in order to analyze the topic of BCT and SCM as objectively as possible. The seven steps of Freels and Onwuegbuzie (2016) serve as the basis of the six steps [22]. For this purpose, the seven steps were interpreted and adapted to the topic. In this section, the procedure shall be explained as comprehensibly as possible.

1. Development of a thorough understanding of the topic;

2. Identification of two main topics, a subject area and subsequent definition of keywords;

3. Performing a preliminary research in selected databases;

4. Performing the research in several available/suitable databases;

5. Conducting a prior evaluation of the quality of the literature;

6. Performing a detailed analysis and classification of the selected literature.

In order to identify two main topics and a subject area, a thorough understanding of the topic is necessary. After that, the keywords needed for the search are defined. By conducting a preliminary search in selected databases, the keywords can be tested and adjusted, if necessary. The actual research was then carried out at Science Direct (SD) and Web of Science (WoS). Based on the titles and, if applicable, the abstracts, the 
literature was evaluated thematically; search terms and impact factors were also used for this purpose. Thereafter, the exploration phase of the literature analysis-the so-called literature research-is completed and the interpretation or analysis of the literature found is begun. The final literature found can subsequently be analyzed and classified in detail.

\subsection{Preparation of the Literature Analysis-Defining the Keywords}

Exploration of the subject matter is a prerequisite for formulating targeted search terms and thus initiating the literature research. In this phase, the title for this literature analysis was worked out and a research question was posed. Then the work here was divided into subtasks. The focus of the analysis has already been defined in the first section of this paper. The aim is to answer the research questions mentioned above with the help of the systematic literature analysis. In addition, an overview of the current state of the art of the possible applications of BCT in the SCM is to be provided.

In order to answer the research questions, sources have to be identified that deal with BCT as well as logistics and SCM. The following three themes are relevant for this paper. First is the BCT, the focus of this paper. Since there is disagreement in the literature about the exact wording of this term, different variations were searched for ("Blockchain", "Block Chain" as well as "Block-Chain"). Some authors also use the term "distributed ledger." It denotes the underlying technology for which the BC is an example. Second, advantages or possible improvements that the implementation of the BCT may be able to provide are the focus of research. The third complex of themes refers to the use cases of BCT. The examined application area of BCT is logistics or SCM. Therefore, "Supply Chain" and "Logistics" were used as search terms. Sources containing the term "Supply Chain Management" were found from the first term. For each complex of themes, technical terms were derived, which in turn were transformed into keywords (see Table 1).

Table 1. Definition of the keywords based on three complexes of themes.

\begin{tabular}{|c|c|c|}
\hline Complex of Themes & Technical Terms & Search Term \\
\hline Blockchain Technology & $\begin{array}{l}\text { Blockchain } \\
\text { Distributed Ledger }\end{array}$ & $\begin{array}{l}\text { "Blockchain", "Block-Chain", "Block Chain" } \\
\text { "Distributed Ledger" }\end{array}$ \\
\hline $\begin{array}{l}\text { advantages or possible } \\
\text { improvements }\end{array}$ & $\begin{array}{l}\text { advantage, advantages } \\
\text { economics, economical, } \\
\text { economically ... } \\
\text { process, processes } \\
\text { challenge. Challenges } \\
\text { problem, problems } \\
\text { example, case study, project }\end{array}$ & $\begin{array}{l}\text { "advantage*" } \\
\text { "econom*"; }(*) \text { represents any group of } \\
\text { character, including no character } \\
\text { "process" } \\
\text { "challeng" } \\
\text { "proble" }\end{array}$ \\
\hline Logistics & $\begin{array}{l}\text { Supply Chain } \\
\text { Supply Chain Management } \\
\text { Logistik, Logistic, Logistics }\end{array}$ & $\begin{array}{l}\text { "Supply Chain" } \\
\text { "Logisti*" }\end{array}$ \\
\hline
\end{tabular}

The search terms of the themes were each linked with the Boolean operator OR. The actual complexes of themes were linked with the Boolean operator AND. The query used for WoS is the following: "TS = (("Blockchain" OR "Block-Chain" OR "Block Chain" OR "Distributed Ledger") AND ("advantag*" OR “econom*” OR "proces*" OR "challeng*" OR "proble*") AND ("Supply Chain" OR "Logisti*"))".

\subsection{Selection of the Relevant Literature}

In this step of the systematic literature analysis, the publications relevant for this work are filtered out manually, out of all the publications found. The search was conducted on 24 May 2021 on the databases SD and WoS. Since SD produced significantly fewer hits on the topics of BCT and SCM, due to the limitations of SD, only these two topics were combined (Note: Science Direct did not support wildcards—status 2021). Therefore, the query for SD has been changed as follows: Title, abstract, keywords: ("Supply Chain" OR "Logistic" OR “Logistics”); Title: ("Blockchain" OR “Block-Chain" OR “Block Chain" OR "Distributed Ledger"). Overall, a preliminary result set of 440 publications was found. 
First, all publications were removed if they were duplicates or not written in either German or English. Subsequently, both the titles and the abstracts of the other sources were evaluated. With the help of this information, each source was assigned a score. The title and the abstract were evaluated in terms of their relevance to the topic of this paper. Furthermore, the literature was evaluated thematically. An indication of quality was provided by the ranking of the journals on various journal ranking platforms (Scimago Journal and Country Rank and the ranking of VHB). The literature selected was evaluated on a scale from 1 to 10 . All sources with a rating below 5 were immediately excluded. A score of 10 was not assigned, which means that no paper was found that perfectly answered the research question. This resulted in a total of 32 relevant publications (see Figure 2).

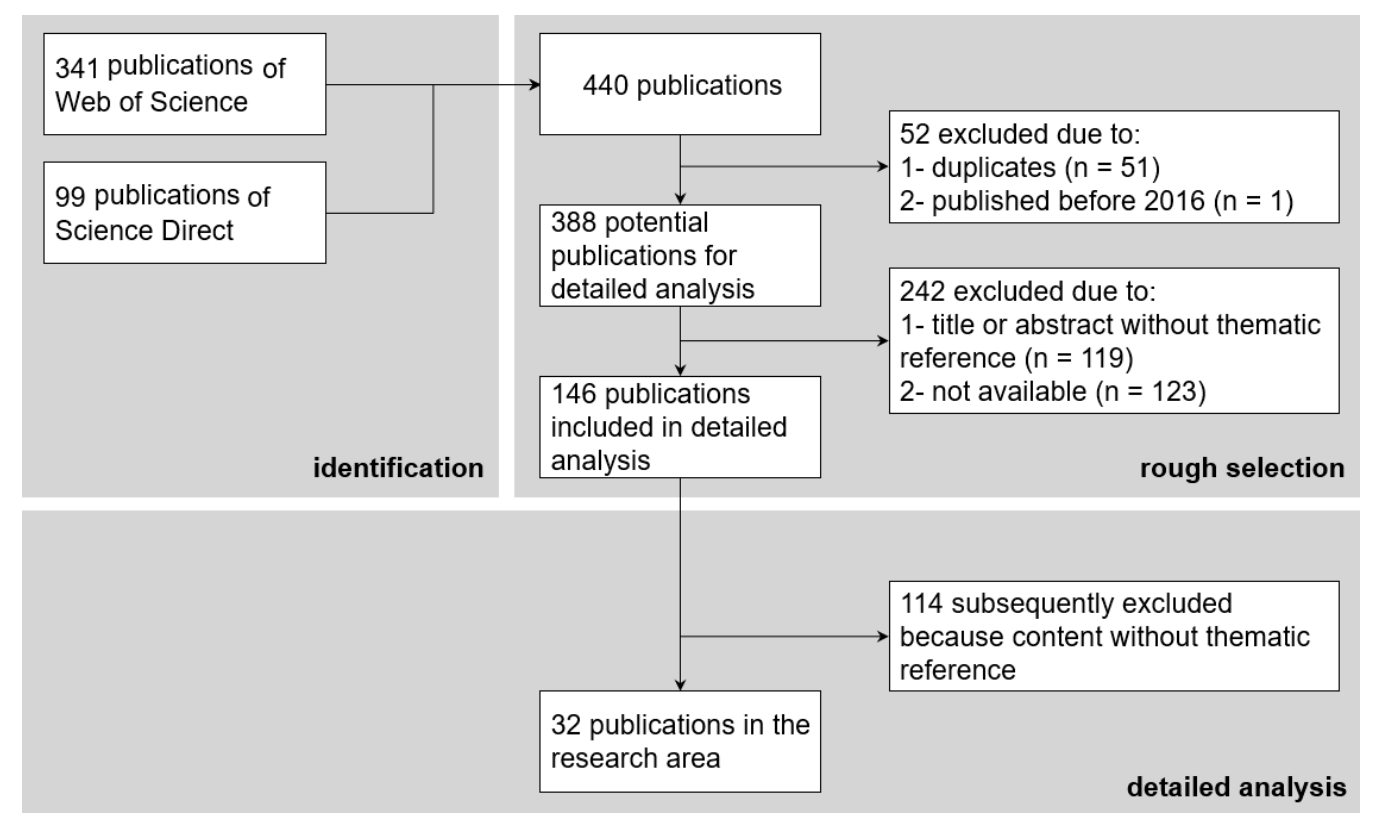

Figure 2. The literature filtering process [23] (p. 3) (greatly modified).

\subsection{Analysis and Synthesis of the Identified Literature}

As already described, the aim of the systematic literature analysis was to identify economic benefits of applying BCT in SCM, as well as real-world examples.

For this purpose, the relevant publications were first synthesized into thematically related clusters (see Figure 3). To form these clusters, the bottom-up approach was applied. After a first analysis of the titles, as well as the abstracts of the publications, two superordinate clusters were identified.

The first group of sources generally deals with the functionalities of BCT. The conditions under which a BC can be used in SCM were discussed. These sources also deal with the advantages and disadvantages of the BC and the adoption barriers. This cluster is referred to as "Overview of BCT in SCM." A more detailed analysis of this cluster is provided in Section 4. The second cluster consists of sources in which the authors deal with the concrete application possibilities of the BCT. This group can be divided into three subclusters: Food SC, Healthcare SC and descriptions of logistics processes within the luxury SC. In Section 5, these application possibilities are analyzed in more detail. In addition, an exemplary logistics process will be described. Due to the high number of publications, the use cases "Food SC" and "SC in Healthcare" are considered separately. One application case will be presented for each of the two clusters in which BCT is already in use. 


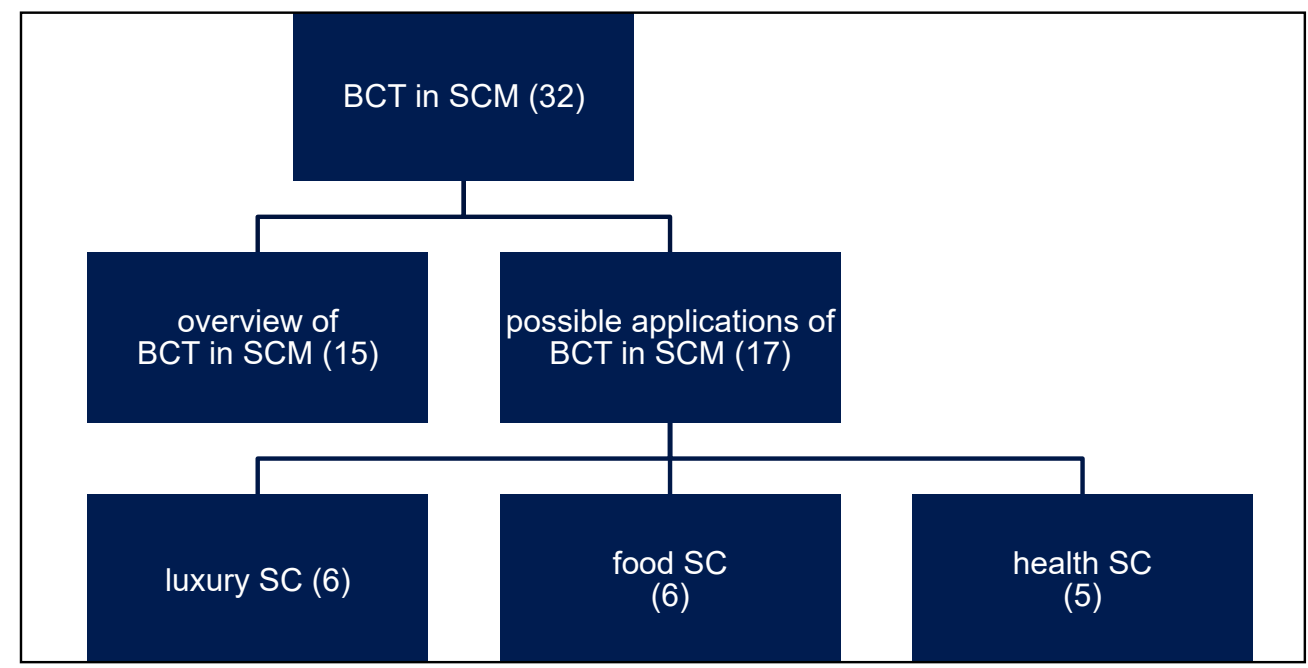

Figure 3. Number of relevant publications in the distinguished clusters.

\section{Blockchain Technology in Supply Chain Management}

In this section, it will be demonstrated which features the BCT can offer to the SCM and which of them are relevant for the SCM. The implementation of the BCT will be explained and the related weaknesses of the BCT will be pointed out. Therefore, the first cluster

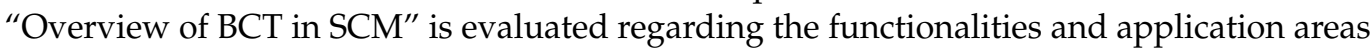
of the BCT in SCM. Subsequently, the barriers and weaknesses of BCT and the factors to be considered when implementing the $\mathrm{BC}$ will be discussed. Finally, from the knowledge gained, the conditions are derived under which the use of a BC in the SCM makes sense. It is already known that $\mathrm{BCT}$ can provide more than just the two functionalities discussed below. However, the paper deliberately focuses on the two functionalities that have the highest potential for the SCM. Wang et al. (2019) name the general advantages of BCT in SCM as first, the improved transparency of SC, second the creation of trust and secure information exchange and third the improvement of processes [3] (p. 226), [24] (p. 34993).

\subsection{Functionalities of the Blockchain Technology in Supply Chain Management}

The possible applications of BCT are very diverse. However, for a meaningful economic use, this technology must solve existing problems of SCs or lead to process improvements. From the analyzed literature of the first cluster "Overview of BCT in SCM," two fundamental functionalities of the BCT have been identified which may be beneficial for SCM. The two identified functionalities of the BCT that have a high potential for the SCM are its functionality as a transparent database and the application of Smart Contracts written on the BC.

\subsubsection{BCT with the Functionality of a Database}

For the first functionality, the $\mathrm{BC}$ acts as a database, whereby the data must be stored permanently and without contradiction along the SC. In this way, a complete, crosscompany data and information flow along the entire SC can be ensured. Thus, the SC can be strengthened, since functional problems of the SC can be found or searched for in a targeted manner. Because nowadays it is not only individual companies that compete with each other, but entire SCs, a SC can generate or secure a competitive advantage over other $\mathrm{SC}$ by increasing its efficiency. Since the data in the BC cannot be manipulated by the participants at a later time, the individual companies along the $\mathrm{SC}$ can gain an advantage because cooperation is more transparent and therefore fairer [25] (p. 5). When acting as a database in SCM, there are two possible advantages that BCT can provide: on the one hand, BCT can provide process improvements and, on the other hand, BCT can offer added value to the customer [26]. In the first case, competitive advantages arise from lower costs and in the second case, customers are willing to pay a higher price. 
With regard to process improvements, it can be stated that a database based on BCT differs from a conventional database in that each participant in the network may have a complete copy of the database. In this way, everyone can copy the current changes to his database and check if it is consistent with the previous blocks [25] (p. 2). BCT is a suitable solution when trust is required and transparency needs to be created. According to Giungato and colleagues (2017), this may be the most important advantage of BCT in SCM and could be applied in many areas of SCM [27] (p. 8). Tijan and colleagues (2019) point out that the developments around Industry 4.0 already offer many possibilities to improve processes that can be supported or enabled by BCT. The goods remain traceable along the entire SC for all participants. Applying Big Data is a necessity for Industry 4.0. Big Data and BCT can benefit each other, because with BCT the data can be collected along the SC, which generates deeper insights through Big Data. This includes a large amount of structured, but also unstructured, data that are often only collected in order to obtain more data. The amount of this data is growing exponentially [7] (p. 6).

Traceability of processes or goods can be of great interest for SCs. Traceability can be implemented on the basis of BCT, which is why Business Process Management conducts research in the area of BCT. Due to the high degree of specialization of companies, SCs are becoming more and more complex and contain more participants, which can lead to trust problems in certain processes within an SC. This is especially problematic if critical goods, such as pharmaceuticals, are part of the SC; regulations and laws require full traceability of origin and processing for some critical goods [28] (p. 56). BCT in SCM could also prevent time delays and significantly reduce human error. Every transport within the SC can be documented reliably with BCT. In the best case, this traceability starts with the mining of the respective raw materials and ends with the purchase by the end customer [7] (p. 6).

The second possibility of BCT generating a competitive advantage in SCM is to offer the customer added value. The increase in transparency does not only provide an advantage for the SC companies, but can also create more trust in the product among final customers [29] (p. 2125). This increased trust could lead to higher customer satisfaction [4] (p. 264) and greater willingness to pay, especially for critical products such as food or pharmaceuticals [30] (p. 36505).

\subsubsection{BCT with the Functionality of Smart Contracts}

Smart Contracts have been identified in the literature as a second way in which BCT could provide advantages in SCM. In Section 2.4, Smart Contracts were presented theoretically. In summary, Smart Contracts are automated, secure contracts that are written to the $\mathrm{BC}$ and only trigger a transaction under previously known conditions. No support of a centralized execution authority is necessary. Smart Contracts enable the automation of complex multi-step processes [12] (p. 2301). For these reasons, the functions of Smart Contracts are very relevant to SCM. Traditional contracts often require a central, trusted third party. This trusted third party often charges high transaction fees and can become a weak point in the process; failure of this party can lead to security problems and a cancellation of the transaction. Furthermore, the decision of a trusted third party is not always objective and understandable for all the parties involved. Smart Contracts are different in that they function as autonomous actors and their behavior is completely predictable [12] (p. 2297).

Smart Contracts come with many problems, which is one reasons why there are hardly any real applications of Smart Contracts in SCM in the literature. The problems can be divided into four categories, which are described in more detail in the next section: programming, security, data protection and performance problems [18] (p. 94).

\subsection{Implementation of Blockchain Technology in Supply Chain Management-Barriers and Weaknesses}

To implement a BC in an SC, it is important that all parties involved agree on a BC and that everyone is involved in the process of implementation. As the average number of participants in a SC has increased, reaching an agreement is becoming more and more 
important, but also more complex and difficult. Some companies gain a greater advantage from the implementation of BCT than others. Therefore, many decisions have to be taken together by the participants. A public BC is the most secure and has the highest transparency and can therefore offer the end customers the best and most trustworthy information. With a public BC, sensitive company data also becomes public. This extensive disadvantage overrides the advantages of a public BC for most companies. In most cases, a private, permissioned BC is used to which only certain parties have access. Only a small part of the data is subsequently released to the final customer [15] (p. 6).

One problem with Smart Contracts is that the legal enforceability is still limited. The problem is made worse by the fact that Smart Contracts in SCM are supposed to be valid across national borders. Efforts are being made to make the technical rules of smart contracts legally enforceable and binding for all parties. As an interim solution until these problems are globally solved, a so-called "Dual Integration" is proposed (See: https: / / erisindustries.com/components / erislegal/ (Last accessed on 7 June 2021)). This involves drawing up two contracts between companies that refer to each other. On the one hand, a real contract is drawn up that is legally binding for all parties. On the other hand, a Smart Contract is drawn up which refers to the real contract between the companies.

If a function of the Smart Contract contains errors (logical or content-related), the decisions made in the Smart Contract cannot be reversed, which is problematic. For example, a Smart Contract in its simplest form can function as a lockbox. It may happen that depositing money into this safe deposit box is possible without any problems, but there is an error in the code for the payment of the funds or the "withdrawal function" (See: https:/ / solidity.readthedocs.io/en/latest/introduction-to-smart-contracts.html (Last accessed on 7 June 2021)). This means that the deposited crypto-currencies are irrecoverably lost and cannot be recovered by any party [12] (p. 2300).

The problems of Smart Contracts can be classified into the following four categories: programming, security, data protection and performance problems. Programming Smart Contracts requires accurate contracts, but programming them is a challenge, because the contracts cannot be changed or cancelled. Another challenge is the complex programming language. Smart contracts should be more secure than traditional contracts that are monitored by third parties. Unfortunately, there are still conceptual problems that might affect security. There is a dependency on time stamps; at Ethereum, for example, the execution of the contract may depend on Miner if two dependent transactions update the same Smart Contract in the same block. Other problems include transaction dependency, criminal activities and untrusted data feeds [18] (p. 94).

Smart Contracts, due to their transparency, give away more information than traditional contracts. The problem becomes more manageable through private BCs, but it still remains. If transparency becomes limited for the protection of company data, the security and trustworthiness of the data is simultaneously reduced. With BCT, the number of executable transactions or Smart Contracts is limited. This can be particularly problematic when executing Smart Contracts sequentially, since with BCT one contract is executed after another. The BCT is only limited and can be scaled at high expense, which can lead to performance problems if the number of contracts to be executed is very high [31] (p. 183).

In summary, BCT is a secure, transparent and decentralized database for monitoring the product flow. The increased transparency can either offer added value to the end customer, or it can help to comply with regulations and laws and provide the companies along the SC with opportunities to increase the efficiency of the SC. The greatest economic potential results from the tracking of critical goods, such as food or pharmaceuticals. In order to make tracking easier for Smart Contracts and to automate the processes, sensors can be used to document the transport of the goods. Due to sensible company data, private, permissioned BCs are better suited than more secure, and more transparent, public BCs. 


\section{Applications of Blockchain Technology in Supply Chain Management}

This paper aims to present real applications of BCT in SCM. First, a logistic process is described and then two use cases are presented, where BCT is already in use. The specific added value of the $\mathrm{BCT}$ for the respective use cases will be shown. In order to achieve this goal, the second cluster "Possible applications of BCT in SCM" will be analyzed. This consists of 17 publications in which the authors deal with the practical applications of this technology in SCM, and whose evaluation resulted in a multitude of different applications. The majority of the publications in the second cluster examine an application of BCT in the food SC (six publications) and an implementation in the SC of the healthcare sector (five publications). For these two major clusters, a concrete use case, where BCT is already in service, will be given. BCT is already used in many areas (e.g., financial engineering and crypto-currencies), but virtually no financially self-sustaining applications exist in the real world [32] (p. 10).

\subsection{An Examplary Logistics Process in Detail-Inside the Luxury Supply Chain}

In the literature, there are various ways that BCT can support a so-called Luxury SC. A current application is the authentication and certification of diamonds, on the one hand to prevent the acquisition of so-called "blood diamonds." On the other hand, conventional certificates can be faked, or a real certificate can be used for a fake diamond, since BC certificates are permanent [33] (p. 17).

Platforms like Everledger offer a unique digital thumbprint for high value and hardto-replace goods, such as diamonds and fine wine [15] (p. 9). This thumbprint is made by using the BCT that is unalterably stored in the BC; the thumbprint consists "of 40 metadata points, the laser inscription on the girdle, and the stone's four Cs-color, clarity, cut, and carat weight." (See: https:/ /www.altoros.com/blog/a-close-look-at-everledgerhow-blockchain-secures-luxury-goods / (Last accessed on 7 June 2021); Primary source: https: / / youtu.be/GAdjL-nultI? $\mathrm{t}=202$ (Everledger, Last accessed on 7 June 2021)).

In addition to the already existing BC-certificates [33] (p. 27), the transport of the diamond could be documented completely by means of the BCT (tracked and traced). The TrustChain ${ }^{\mathrm{TM}}$ from IBM can already track and authenticate diamonds [34] (p. 587) (Source: https: / / www.trustchainjewelry.com (Last accessed on 7 June 2021)). Transports usually involve several transshipments (processes in which the mean of transport is changed). The diamond is transported by different transport service providers. Ideally, the diamond would have to be checked for its digital thumbprint at each transfer point and the result would have to be stored in the BC. For one thing, checking the diamond takes time and for another, special measuring technology is required.

One way to speed up the process is to mandate that only the final receiver checks the digital thumbprint. The seller and the sender are responsible for the quality of the diamond, and can authenticate and certify it using the BCT. The shipper seals a transport box and is responsible for the fact that the diamond matching the digital thumbprint is in the box. Inside the box, there is an RFID chip beside the diamond, and a barcode is attached to the outside of the box. Inside the box, further sensors can be installed, which can be connected (e.g., via Bluetooth), such as temperature sensors or acceleration sensors, which might be able to detect shocks [34] (p. 587). The shipment could be refused on the basis of the sensor data.

In this example, the diamond is transported as follows: from the shipping point, the diamond is transported by truck to the freight port by a forwarding agent. There the diamond is transshipped into a cargo ship; at the final port, the diamond changes the means of transport (from the ship to the truck) and the responsible party. The truck then transports the diamond directly to the recipient. During the actual transport, the diamond can be shipped in a properly sealed package (e.g., a hard-plastic box). Opening the box is thus visible to the person next in the transport chain. The condition of the package is documented with each handling and saved in a block of the BC. For this purpose, the following transfer protocol could be processed for each transfer: 
- Is the package intact and in a flawless condition?

- Is the seal undamaged?

- Do the barcode and RFID match the shipping note?

- Confirmation that the package has been passed.

As soon as the recipient of the package confirms that the package has been delivered, a transaction can be triggered by means of a Smart Contract. One advantage of BCT is that it can be used across different companies, which means that the transportation means of different companies can be used, as long as they agree to use the same BC. No one can change the data in the $\mathrm{BC}$ retrospectively. $\mathrm{As}$ the $\mathrm{BC}$ is law, the currently responsible party for the diamond is always visible to all parties; this currently responsible party is liable for any damage or loss. The responsible persons can individually insure themselves. In the case of a public block certificate, there is a risk that criminals could gain access to the current location of the valuable diamond, which makes a private, permissioned $\mathrm{BC}$ suitable [15] (p. 6). However, a residual risk remains because all companies along the SC have access to the $\mathrm{BC}$.

\subsection{Food Supply Chains}

Although the literature analysis found quite a number of publications in the field of food SCs, no case was found in which a company actively used the BCT to earn money and gain a competitive advantage. Many feasibility studies have been found, but only few have tested the prototypes in the real world; this finding matches the findings of Hinckeldeyn (2019) [16] (p. 32). In the following section, several projects are briefly mentioned as examples, followed by a more detailed description of one application.

First, there is the cooperation of Walmart and Hyperledger Fabric, a BC platform for companies, which is intensively supported by IBM [16] (p. 24) and has been found referenced numerous times (See: https: / fortune.com/2017/08/22/walmart-blockchainibm-food-nestle-unilever-tyson-dole/ (Last accessed on 7 June 2021)). In a field test, Walmart and IBM were able to demonstrate that the origin of mangoes and pork could be determined via BC within a short time [16] (p. 32). The IBM Trustchain can track tomatoes from the farm to the pot, to the jar, to the table, but is not yet in use, although a working prototype is (Source: https: / / www.trustchainjewelry.com (Last accessed on 7 June 2021)). Bumble Bee Foods cooperates with SAP to document tuna fish products from Indonesia. The size of the fish, fishing location and time, freshness during its processing and the company's certificate of production are stored within a BC (See: https: / cointelegraph.com/ news/north-american-seafood-firm-to-use-blockchain-tech-in-supply-chain/amp (Last accessed on 7 June 2021)). Other examples deal with the traceability and certification of Extra Virgin Olive Oil [35] (p. 173) or describe how eggs can be traced in the USA using BCT [36] (p. 1).

On the one hand, these examples demonstrate the increased interest of food manufacturers in the trustworthy documentation of their products in a BC [37]. Many companies are already trying to gain the trust of consumers with the help of seals, such as the Fairtrade label, which is given by a Fairtrade organization under certain conditions. On the other hand, the examples also show the interest of the large IT groups. Whether the interest comes from the Research and Development department or the Marketing department is not always identifiable. Food scandals have led to manufacturers generating competitive advantages by providing reliable proof of the origin and processing of goods. The BCT might be able to strengthen the confidence of the consumer [16] (p. 32).

In the following section, a prototype that has been tested in the field will be discussed. Zhang and colleagues (2020) have developed a new system architecture along the entire Grain SC based on BCT. Compared to traditional methods of the SCM, their system is characterized by high data security, real-time exchange of relevant information (such as hazardous material information) and trustworthy grain tracing along the entire SC [38] (p. 36398). 
The Grain SC starts with grain cultivation and production, primary grain processing, grain product cycles, and deep grain processing and ends with transport to the consumer (see Figure 4). Zhang and colleagues (2020) have identified five typical links in the traditional Grain SC (see Figure 4): the link of grain production (G1), the link of grain storage (G2), the link of grain processing (G3), the link of grain logistics and transport (G4) and the link of grain marketing (G5) [38] (p. 36400).

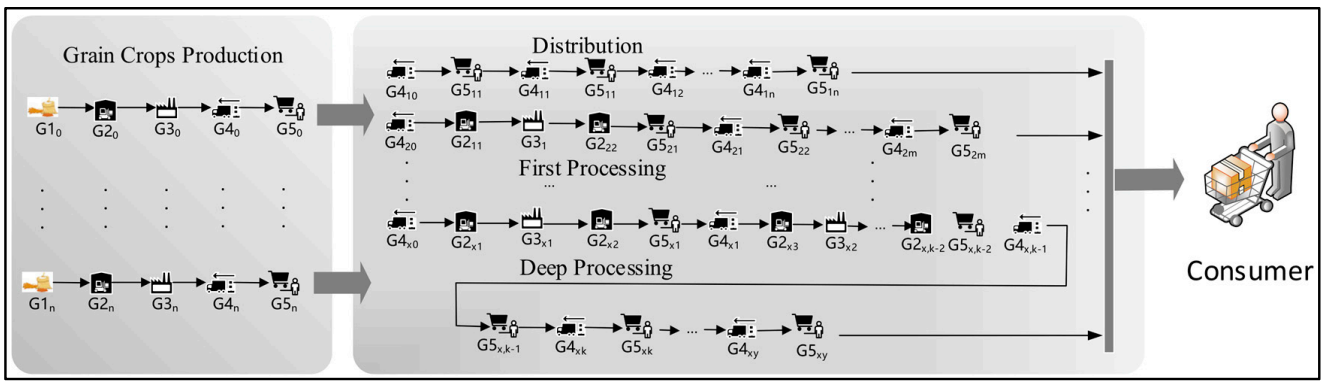

Figure 4. Structure of the Grain Supply Chain [38] (p. 36401).

Smart Contracts define the conditions for the execution of the transactions in advance. The system collects data about the grain, which is then stored within the BC. The data are collected mainly by electronic tags and various sensors such as code-scan guns, cameras, smoke detectors, humidity sensors, light sensors, etc. When the parameters of the grain meet the requirements and all other requirements are met, the transaction is automatically triggered [38] (p. 36404).

A special use case was applied to validate the proposed system. An Information Management System for the Grain SC was established with grain companies in the province of Shandong. This field test was conducted on the BC platform of Hyperledger Fabric with the cloud database MySQL. The system was able to provide reliable information in the SC for participants, consumers and third parties, and the data provided a good basis for assessing, predicting and early warning of hazards [38] (p. 36407).

\subsection{Supply Chains in Healthcare}

The company Modum, a Swiss start-up, is to be shown as an example for SCs in Healthcare. Modum has initiated several studies and has developed and tested prototypes and offers its services in the field of BCT in SCM. The goal of the company is the secure tracking and tracing of pharmaceutical products. To this end, the company relies on the public Ethereum BC. For tracking, IoT sensor devices (IoT = Internet of things), QR codes and barcodes are used to clearly identify the items handled.

The Good Distribution Practice Regulation (GDP 2013/C 343/01) requires proof that the transport conditions (in particular the temperature) could not have affected the quality of the pharmaceutical products transported [39]. The company Modum.io enables other companies to meet the GDP requirements with the help of BCT. At the same time, it should be possible to generate significant cost savings for the transport of pharmaceuticals that do not require active cooling [40] (p. 5). The system is based on the components shown in Figure 5 . 


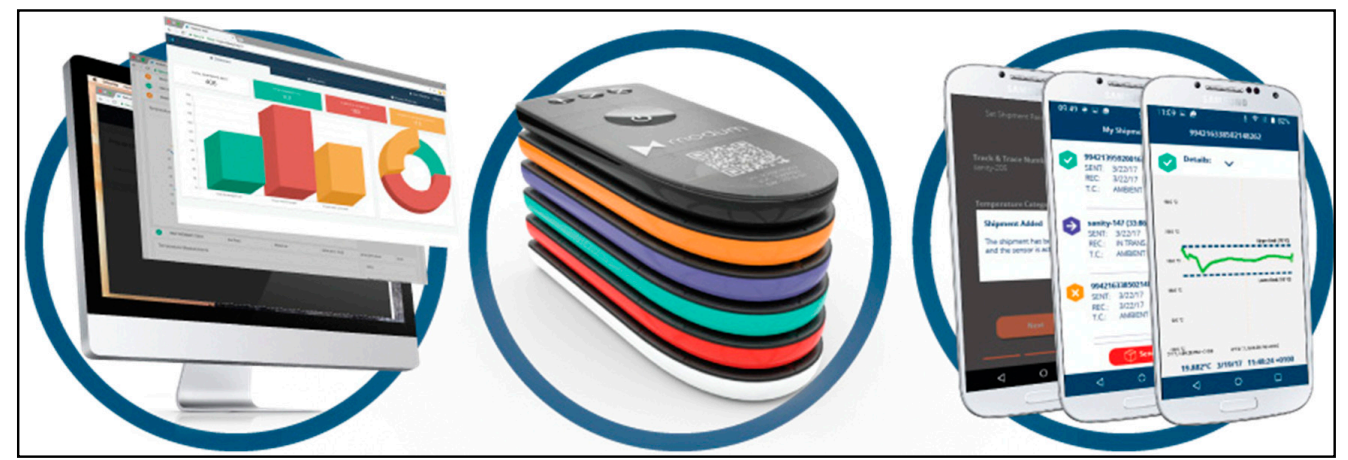

Figure 5. Components of the system from Modum are a dashboard, a temperature logger and a mobile application [40] (p. 6).

To monitor the temperature during the transport of medicines, a calibrated temperature sensor in the package stores a measured value every $10 \mathrm{~min}$. When receiving the package, the customer can scan the ID number on the package and then request the temperature data via Bluetooth, without having to open the package [40] (p. 5). The customer then sends the data to the Smart Contract (see Figure 6). The data are stored in PostgreSQL because the collected data is too large or too sensitive to be stored in the Ethereum BC [34] (p. 588).
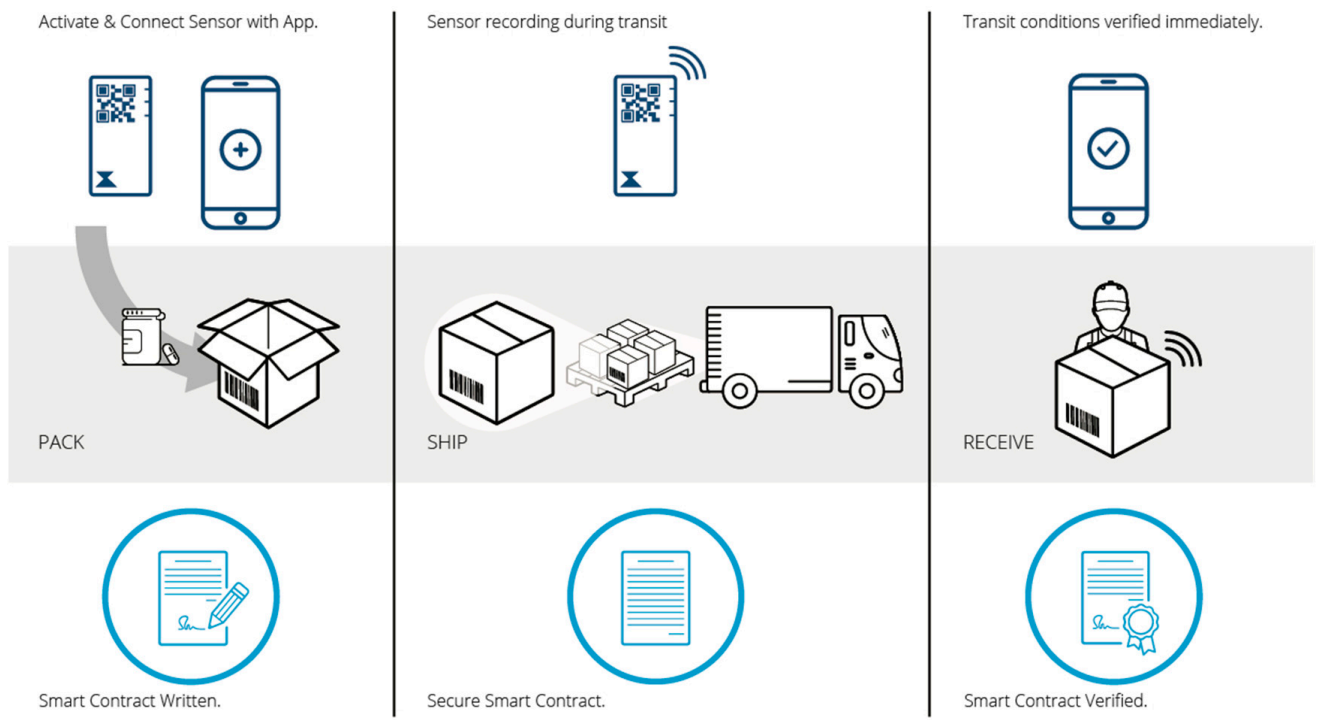

Figure 6. Logistics process of the company Modum [40] (p. 8).

\section{Conclusions and Outlook}

This work aimed to identify the economic benefits of implementing BCT in logistics by means of a structured literature review. In order to do this, this paper introduced the BCT, described its origins in Bitcoin and the structure and core properties of a BC. A comprehensive and structured literature analysis was performed to identify concepts for the use of BCT in logistics in terms of economic benefits. The cluster "Possible applications of BCT in SCM" and the sub-clusters of Food and Healthcare SC were identified, among others. In addition to two concrete use cases, an exemplary logistics process was presented, showing the advantages of the BCT for each individual process step.

Based on this study, new insights into the added value of BCT can be perceived. Companies that do not have expertise in BCT can use this paper to assist them in their technology selection. In addition, this paper provides managerial insights by identifying current possible applications of the technology, examining them for their benefits, and thus highlighting possible research gaps. The applications found highlight opportunities and can inspire companies. 
In summary, the main advantages of a $\mathrm{BC}$ are that, unlike traditional distributed databases, no intermediary or central point of account is required, because the participants in the network control each other. In this way, the $\mathrm{BC}$ creates a consensus on the current state of the network without requiring the individual partners of the SC to trust each other. It also guarantees the integrity and immutability of the information stored on it.

The BCT is considered a new technology with many weaknesses and adoption barriers. The main feature is the decentralized structure, which, in combination with transparency, sets new security standards. Compared to conventional central databases, BCT is quite complex, and the high level of security is paid for with high hardware and energy costs. This effort is only worthwhile if the advantages resulting from the transparency can outweigh the disadvantages. For critical goods, such as food or pharmaceuticals, or in luxury SC, the BCT could be used more and more frequently.

A finding of the study is that BCT is particularly useful for goods with a high value. In addition, the advantages of $\mathrm{BCT}$ unfold best when the trading volume of the respective goods is low. The demand for transparency and immutability of data must be more important than the protection of sensitive data. This work has some limitations which should be considered when looking at the results. Initially, two databases were used in the systematic literature analysis. Therefore, it may happen that some relevant papers were not found.

Author Contributions: Conceptualization, M.B. and H.W.; methodology, M.B., D.B.; software, M.B.; validation, M.B., D.B. and H.W.; formal analysis, M.B.; investigation, M.B.; resources, M.B.; data curation, M.B.; writing-original draft preparation, M.B.; writing-review and editing, M.B.; visualization, M.B.; supervision, H.W. All authors have read and agreed to the published version of the manuscript.

Funding: This research received no external funding.

Data Availability Statement: Data sharing not applicable.

Acknowledgments: The authors thank the anonymous reviewers for their constructive support. The comments helped to improve the quality of the paper.

Conflicts of Interest: The authors declare no conflict of interest.

\section{References}

1. Nakamoto, S. Bitcoin: A Peer-to-Peer Electronic Cash System. 2009, p. 9. Available online: https://bitcoin.org/bitcoin.pdf (accessed on 21 June 2021).

2. Dujak, D.; Sajter, D. Blockchain Applications in Supply Chain. In SMART Supply Network; Kawa, A., Maryniak, A., Eds.; Springer International Publishing: Cham, Germany, 2019; pp. 21-46.

3. Wang, Y.; Singgih, M.; Wang, J.; Rit, M. Making sense of blockchain technology: How will it transform supply chains? Int. J. Prod. Econ. 2019, 211, 221-236. [CrossRef]

4. Petersen, M.; Hackius, N.; von See, B. Mapping the sea of opportunities: Blockchain in supply chain and logistics. Inf. Technol. 2018, 60, 263-271. [CrossRef]

5. Nakano, M. Supply Chain Management: Strategy and Organization; Springer: Singapore, 2020.

6. Kaluza, B.; Winkler, H. Management and Controlling of Supply Chains. Int. J. Appl. Econ. Econom. IJAEE 2005, 13, 247-262.

7. Tijan, E.; Aksentijević, S.; Ivanić, K.; Jardas, M. Blockchain Technology Implementation in Logistics. Sustainability 2019, 11, 1185. [CrossRef]

8. Yli-Huumo, J.; Ko, D.; Choi, S.; Park, S.; Smolander, K. Where Is Current Research on Blockchain Technology?-A Systematic Review. PLoS ONE 2016, 11, e0163477. [CrossRef]

9. Kim, J.-S.; Shin, N. The Impact of Blockchain Technology Application on Supply Chain Partnership and Performance. Sustainability 2019, 11, 6181. [CrossRef]

10. Antonopoulos, A.M. Mastering Bitcoin: Programming the Open Blockchain, 2nd ed.; O'Reilly: Sebastopol, CA, USA, 2017.

11. Zheng, Z.; Xie, S.; Dai, H.; Chen, X.; Wang, H. An Overview of Blockchain Technology: Architecture, Consensus, and Future Trends. In Proceedings of the 2017 IEEE International Congress on Big Data (BigData Congress), Honolulu, HI, USA, 25-30 June 2017; pp. 557-564. [CrossRef]

12. Christidis, K.; Devetsikiotis, M. Blockchains and Smart Contracts for the Internet of Things. IEEE Access 2016, 4, $2292-2303$. [CrossRef] 
13. Xu, X.; Weber, I.; Staples, M.; Zhu, L.; Bosch, J.; Bass, L.; Pautasso, C.; Rimba, P. A Taxonomy of Blockchain-Based Systems for Architecture Design. In Proceedings of the 2017 IEEE International Conference on Software Architecture (ICSA), Gothenburg, Sweden, 3-7 April 2017; pp. 243-252. [CrossRef]

14. Novo, O. Blockchain Meets IoT: An Architecture for Scalable Access Management in IoT. IEEE Internet Things J. 2018, 5, 1184-1195. [CrossRef]

15. Chung, G.; González-Peralta, J.; Turner, K.; Gockel, B. Blockchain in Logistics-Perspectives on the Upcoming Impact of Blockchain Technology and Use Cases for the Logistics Industry. 2018, p. 28. Available online: https://www.dhl.com/content/dam/dhl/ global/core/documents/pdf/glo-core-blockchain-trend-report.pdf (accessed on 21 June 2021).

16. Hinckeldeyn, J. Blockchain-Technologie in der Supply Chain: Einführung und Anwendungsbeispiele; Springer Fachmedien Wiesbaden: Wiesbaden, Germany, 2019.

17. Ammous, S. The Bitcoin Standard: The Decentralized Alternative to Central Banking; Wiley: Hoboken, NJ, USA, 2018.

18. Abdelhamid, M.; Hassan, G. Blockchain and Smart Contracts; ACM: New York, NY, USA, 2019; pp. 91-95.

19. Bagloee, S.A.; Tavana, M.; Withers, G.; Patriksson, M.; Asadi, M. Tradable mobility permit with Bitcoin and Ethereum-A Blockchain application in transportation. Internet Things 2019, 8, 100103. [CrossRef]

20. Szabo, N. Formalizing and Securing Relationships on Public Networks. First Monday 1997, 2. [CrossRef]

21. Kumar, A.; Liu, R.; Shan, Z. Is Blockchain a Silver Bullet for Supply Chain Management? Technical Challenges and Research Opportunities. Decis. Sci. 2020, 51, 8-37. [CrossRef]

22. Frels, R.; Onwuegbuzie, A. Seven Steps to a Comprehensive Literature Review; Sage Publications Ltd.: London, UK, 2016.

23. Moher, D.; Liberati, A.; Tetzlaff, J.; Altman, D.G. The PRISMA Group Preferred Reporting Items for Systematic Reviews and Meta-Analyses: The PRISMA Statement. PLoS Med. 2009, 6, e1000097. [CrossRef] [PubMed]

24. Hackius, N.; Petersen, M. Translating High Hopes Into Tangible Benefits: How Incumbents in Supply Chain and Logistics Approach Blockchain. IEEE Access 2020, 8, 34993-35003. [CrossRef]

25. Niu, X.; Li, Z. Research on Supply Chain Management Based on Blockchain Technology. J. Phys. Conf. Ser. 2019, 1176, 042039. [CrossRef]

26. Babich, V.; Hilary, G. OM Forum-Distributed Ledgers and Operations: What Operations Management Researchers Should Know About Blockchain Technology. Manuf. Serv. Oper. Manag. 2020, 22, 223-240. [CrossRef]

27. Giungato, P.; Rana, R.; Tarabella, A.; Tricase, C. Current Trends in Sustainability of Bitcoins and Related Blockchain Technology. Sustainability 2017, 9, 2214. [CrossRef]

28. Di Ciccio, C. Blockchain-Based Traceability of Inter-organisational Business Processes. In Business Modeling and Software Design Bmsd 2018; Shishkov, B., Ed.; Springer: Berlin, Germany, 2018; Volume 319, pp. 56-68.

29. Roeck, D.; Sternberg, H.; Hofmann, E. Distributed ledger technology in supply chains: A transaction cost perspective. Int. J. Prod. Res. 2019, 58, 2124-2141. [CrossRef]

30. Al-Jaroodi, J.; Mohamed, N. Industrial Applications of Blockchain; Institute of Electrical and Electronics Engineers: Piscataway, NJ, USA, 2019; pp. 550-555.

31. Karamitsos, I.; Papadaki, M.; Al Barghuthi, N.B. Design of the Blockchain Smart Contract: A Use Case for Real Estate. J. Inf. Secur. 2018, 9, 177-190. [CrossRef]

32. Demestichas, K.; Peppes, N.; Alexakis, T.; Adamopoulou, E. Blockchain in Agriculture Traceability Systems: A Review. Appl. Sci. 2020, 10, 4113. [CrossRef]

33. Choi, T.-M. Blockchain-technology-supported platforms for diamond authentication and certification in luxury supply chains. Transp. Res. Part E Logist. Transp. Rev. 2019, 128, 17-29. [CrossRef]

34. Azzi, R.; Chamoun, R.K.; Sokhn, M. The power of a blockchain-based supply chain. Comput. Ind. Eng. 2019, 135, 582-592. [CrossRef]

35. Arena, A.; Bianchini, A.; Perazzo, P.; Vallati, C.; Dini, G. Bruschetta: An IoT Blockchain-Based Framework for Certifying Extra Virgin Olive Oil Supply Chain; Institute of Electrical and Electronics Engineers: Piscataway, NJ, USA, 2019; pp. 173-179.

36. Bumblauskas, D.; Mann, A.; Dugan, B.; Rittmer, J. A blockchain use case in food distribution: Do you know where your food has been? Int. J. Inf. Manag. 2020, 52, 102008. [CrossRef]

37. Barge, P.; Biglia, A.; Comba, L.; Aimonino, D.R.; Tortia, C.; Gay, P. Radio Frequency IDentification for Meat Supply-Chain Digitalisation. Sensors 2020, 20, 4957. [CrossRef]

38. Zhang, X.; Sun, P.; Xu, J.; Wang, X.; Yu, J.; Zhao, Z.; Dong, Y. Blockchain-Based Safety Management System for the Grain Supply Chain. IEEE Access 2020, 8, 36398-36410. [CrossRef]

39. European Union. Guidelines of 5 November 2013 on Good Distribution Practice of Medicinal Products for Human Use Text with EEA Relevance. 2013, p. 14. Available online: https:/ / eur-lex.europa.eu/LexUriServ/LexUriServ.do?uri=OJ:C:2013:343:0001: 0014:EN:PDF (accessed on 21 June 2021).

40. Modum. Data Integrity for Supply Chain Operations Powered by Blockchain Technology, Modum. August 2017. Available online: https:/ / modum.io/wp-content/uploads/2017/08/modum-whitepaper-v.-1.0.pdf (accessed on 19 June 2020). 